Received: 10.08 .2021

Revised: 10.10 .2021

Accepted: 29.10 .2021

DOI: $10.17804 / 2410-9908.2021 .5 .044-059$

\title{
MECHANICAL PROPERTIES AND STRUCTURE FORMATION OF ALUMINUM-SILICON ALLOYS AFTER FRICTION STIR PROCESSING
}

\author{
A. V. Chumaevsky ${ }^{\text {a)* }}$, D. V. Indoitu ${ }^{\text {b) }}$, A. V. Sudarikov ${ }^{\text {c) }}$, A. P. Zykova ${ }^{\text {d) }}$, A. R. Dobrovolsky ${ }^{\text {e) }}$, \\ T. A. Kalashnikova ${ }^{\text {f) }}$, V. E. Rubtsov ${ }^{\text {g) }}$, and E. A. Kolubaev ${ }^{\text {h) }}$ \\ Institute of Strength Physics and Materials Science, Siberian Branch of the Russian Academy of Sciences, \\ 2/4 Akademicheskiy Ave., Tomsk, 634055, Russian Federation \\ a) iD http://orcid.org/0000-0002-1983-4385 tch7av@ gmail.com; \\ b) danil.indoitu@mail.ru; \\ c) iD http://orcid.org/0000-0001-8555-3963 avsudarikov@ispms.tsc.ru; \\ d) iD http://orcid.org/0000-0001-8779-3784 zykovaap@mail.ru; \\ e) (iD http://orcid.org/0000-0001-6872-3721 artdobrov@ispms.tsc.ru; \\ f) iD http://orcid.org/0000-0002-0388-4011 gelombang@ispms.tsc.ru; \\ g) iD http://orcid.org/0000-0003-0348-1869 $ه$ rvy@ispms.ru; \\ h) (iD http://orcid.org/0000-0001-7288-3656 ak @ispms.ru \\ *Corresponding author. E-mail: tch7av@gmail.com \\ Address for correspondence: 2/4, pr. Akademicheskiy, Tomsk, 634055, Russian Federation
}

Tel.: 89618914149

This study presents the results of experimental work on obtaining and investigating samples obtained by multipass friction stir processing of the Al-12 \%Si and Al-9 \%Si aluminum-silicon alloys. The results indicate a beneficial effect of friction stir processing on the strength properties of both alloys. The number of tool passes along the processing line has no effect on the mechanical properties and particle size of silicon in the aluminum matrix. The greatest influence on the mechanical properties is exerted by the first pass with the tool. The yield strength of the processed Al-9 \% Si alloy increases by more than a factor of 1.5 , whereas for the Al-12\% $\mathrm{Si}$ alloy there is no increase in yield strength. The tensile strength of the Al-12\%Si alloy increases by $17 \%$, while that of the Al-9 \% Si alloy increases by $29 \%$. The most significant is the increase in relative elongation to failure, namely $77 \%$ for Al-12\%Si and $113 \%$ for Al-9 \% Si.

Keywords: friction stir processing, Al-Si alloys, tensile strength, relative elongation, hardening of aluminum alloys.

\section{Acknowledgment}

The work was performed according to the Government research assignment for ISPMS SB RAS, project FWRW-2021-0012.

\section{References}

1. Kalashnikova T.A., Gusarova A.V., Chumayevskii A.V., Knyazhev E.O., Shvedov M.A., Vasiliev P.A. Regularities of the formation of materials with a composite structure using additive electron-beam technology, friction stir welding and friction stirring processing. Obrabotka Metallov (Metal Working and Material Science), 2019, vol. 21, no 4, pp. 94-112. DOI: 10.17212/1994-63092019-21.4-94-112. (In Russian). 
2. Mishra R.S., Ma Z.Y. Friction stir welding and processing. Materials Science and Engineering: R: Reports, 2005, vol. 50, pp. 1-78. DOI: 10.1016/j.mser.2005.07.001.

3. Li K., Liu X., Zhao Y. Research Status and Prospect of Friction Stir Processing Technology. Coatings, 2019, vol. 9, iss. 2, pp. 1-14. DOI: 10.3390/coatings9020129.

4. Agrawal P., Haridas R.S., Yadav S., Thapliyal S., Gaddam S., Verma R., Mishra R.S. Processingstructure-property correlation in additive friction stir deposited Ti-6Al-4V alloy from recycled metal chips. Additive Manufacturing, 2021, vol. 47, pp. 102259. DOI: 10.1016/j.addma.2021.102259.

5. Zykova A.P., Tarasov S.Y., Chumaevskiy A.V., Kolubaev E.A. A Review of Friction Stir Processing of Structural Metallic Materials: Process Properties, and Methods. Metals, 2020, vol. 10 (6), pp. 1-35. DOI: 10.3390/met10060772.

6. Ma Z.Y. Friction Stir Processing Technology: A Review. Metallurgical and Materials Transactions A, 2008, vol. 39, pp. 642-658. DOI: 10.1007/s11661-007-9459-0.

7. Kalashnikov K.N., Tarasov S.Y., Chumaevskii A.V., Fortuna S.V., Eliseev A.A., Ivanov A.N. Towards aging in a multipass friction stir-processed AA2024. International Journal of Advanced Manufacturing Technology, 2019, vol. 103, iss. 5-8, pp. 2121-2132. DOI: 10.1007/S00170-019-03631-3.

8. Hsu C.J., Kao P.W., Ho N.J. Intermetallic-reinforced aluminum matrix composites produced in situ by friction stir processing. Materials Letters, 2007, vol. 61, iss. 6, pp. 1315-1318. DOI: 10.1016/J.MATLET.2006.07.021.

9. Dolatkhah A., Golbabaei P., Besharati Givi M.K., Molaiekiya F. Investigating effects of process parameters on microstructural and mechanical properties of $\mathrm{Al} 5052 / \mathrm{SiC}$ metal matrix composite fab-ricated via friction stir processing. Materials and Design, 2012, vol. 37, pp. 458464. DOI: 10.1016/J.MATDES.2011.09.035.

10. Zhao H., Pan Q., Qin Q., Wu Y., Su X. Effect of the processing parameters of friction stir processing on the microstructure and mechanical properties of 6063 aluminum alloy. Materials Science \& Engineering A, 2019, vol. 751, pp. 70-79. DOI: 10.1016/J.MSEA.2019.02.064.

11. Su J.Q., Nelson T.W., Colin S.J. Microstructure evolution during FSW/FSP of high strength aluminum alloys. Materials Science and Engineering, 2005, vol. 405, iss. 1-2, pp. 277-286. DOI: 10.1016/J.MSEA.2005.06.009.

12. Manochehrian A., Heidarpour A., Mazaheri Y., Ghasemi S. On the surface reinforcing of A356 aluminum alloy by nanolayered Ti3AlC2 MAX phase via friction stir processing. Surface \& Coatings Technology, 2019, vol. 377, pp. 124884. DOI: 10.1016/J.SURFCOAT.2019.08.013.

13. Jain V.K.S., Varghese J., Muthukumaran. S. Effect of First and Second Passes on Microstructure and Wear Properties of Titanium Dioxide-Reinforced Aluminum Surface Composite via Friction Stir Processing. Arabian Journal for Science and Engineering, 2019, vol. 44, pp. 949-957. DOI: 10.1007/S13369-018-3312-1.

14. Darzi Bourkhani R., Eivani A.R., Nateghi H.R. Through-thickness inhomogeneity in microstructure and tensile properties and tribological performance of friction stir processed AA1050- $\mathrm{Al}_{2} \mathrm{O}_{3}$ nanocomposite. Composites Part B, 2019, vol. 174, pp. 107061. DOI: 10.1016/J.COMPOSITESB.2019.107061.

15. Satish Kumar T., Suganya Priyadharshini G., Shalini S., Krishna Kumar K., Subramanian R. Characterization of NbC-Reinforced AA7075 Alloy Composites Produced Using Friction Stir Processing. Transactions of the Indian Institute of Metals, 2019, vol. 72, iss. 6, pp. 1593-1596. DOI: $10.1007 / \mathrm{s} 12666-019-01566-7$.

16. Deore H.A., Mishra J., Rao A.G., Mehtani H., Hiwarkar V.D. Effect of filler material and post process ageing treatment on microstructure, mechanical properties and wear behaviour of friction stir processed AA 7075 surface composites. Surface \& Coatings Technology, 2019, vol. 374, pp. 52-64. DOI: 10.1016/J.SURFCOAT.2019.05.048.

17. Zhang S., Chen G., Wei J., Liu Y., Xie R., Liu Q., Zeng S., Zhang G., Shi Q. Effects of energy input during friction stir processing on microstructures and mechanical properties 
of aluminum/carbon nanotubes nanocomposites. Journal of Alloys and Com-pounds, 2019, vol. 798, pp. 523-530. DOI: 10.1016/J.JALLCOM.2019.05.269.https://doi.org/10.1016/j.jallcom.2019.05.269

18. Abrahams R., Mikhail J., Fasihi P. Effect of friction stir process parameters on the mechanical properties of 5005-H34 and 7075-T651 aluminium alloys. Materials Science \& Engineering A, 2019, vol. 751, pp. 363-373. DOI: 10.1016/J.MSEA.2019.02.065.

19. Ramesh K.N., Pradeep S., Pancholi V. Multipass Friction-Stir Processing and its Effect on Mechanical Properties of Aluminum Alloy 5086. Metallurgical and Materials Transactions A, 2012, vol. 43, pp. 4311-4319. DOI:10.1007/s11661-012-1232-3.

20. Indoitu D.V., Gusarova A.V., Zykova A.P., Kalashnikova T.A., Chumaevskii A.V., Gurianov D.A., Beloborodov V.A. Friction Stir Processing Regularities of Cast Aluminum Alloy AlSi12. Journal of Physics: Conference Series, 2021, vol. 1989 (1), pp. 012030. DOI: 10.1088/1742-6596/1989/1/012030.

21. Abbadi M., Hähner P., Zeghloul A. On the characteristics of Portevin-Le Chatelier bands in aluminum alloy 5182 under stress-controlled and strain-controlled tensile testing. Materials Science and Engineering A, 2002, vol. 337, iss. 1-2, pp. 194-201. DOI: 10.1016/S0921-5093(02)00036-9. 
Подана в журнал: 10.08 .2021

УДК 539.25:621.791.14:620.186.12

DOI: $10.17804 / 2410-9908.2021 .5 .044-059$

\title{
ФОРМИРОВАНИЕ СТРУКТУРЫ И МЕХАНИЧЕСКИЕ СВОЙСТВА АЛЮМИНИЕВО-КРЕМНИЕВЫХ СПЛАВОВ ПОСЛЕ ФРИКЦИОННОЙ ПЕРЕМЕШИВАЮЩЕЙ ОБРАБОТКИ
}

\author{
А. В. Чумаевский ${ }^{\mathrm{a}}{ }^{*}$, Д. В. Индоиту ${ }^{6)}$, А. В. Судариков ${ }^{\text {в) }}$, А. П. Зыкова ${ }^{\text {г) }}$, \\ А. Р. Добровольский ${ }^{\text {I) }}$, Т. А. Калашникова ${ }^{\text {e) }}$, В. Е. Рубцов ${ }^{\text {() }}$, Е. А. Колубаев ${ }^{3)}$ \\ Институт физики прочности и материаловедения Сибирского отделения Российской академии наук, \\ 2/4, пр. Академический, г. Томск, 634055, Российская Федераџия \\ a) iD http://orcid.org/0000-0002-1983-4385 tch7av@ gmail.com; \\ б) danil.indoitu@ mail.ru; \\ в) (iD http://orcid.org/0000-0001-8555-3963 avsudarikov@ispms.tsc.ru; \\ г) (iD http://orcid.org/0000-0001-8779-3784 zykovaap@mail.ru; \\ д) (iD http://orcid.org/0000-0001-6872-3721 artdobrov@ispms.tsc.ru; \\ e) (iD http://orcid.org/0000-0002-0388-4011 gelombang@ispms.tsc.ru; \\ ж) (iD http://orcid.org/0000-0003-0348-1869 rvy@ispms.ru; \\ 3) iD http://orcid.org/0000-0001-7288-3656 @ eak@ispms.ru \\ *Ответственный автор. Электронная почта: tch7av@gmail.com \\ Адрес для переписки: пр. Академический 2/4, г. Томск, 634055, Российская Федерация \\ Тел.: 8-961-891-41-49
}

В работе представлены результаты экспериментальной работы по получению и исследованию образцов, полученных методом многопроходной фрикционной перемешивающей обработки алюминиево-кремниевых сплавов АК12 и АК9. Полученные результаты свидетельствуют о положительном влиянии на прочностные показатели обоих сплавов фрикционной перемешивающей обработки. Количество проходов инструментом вдоль линии обработки не значительно влияет на механические свойства и размер частиц кремния в алюминиевой матрице. Наибольшее влияние на механические свойства оказывает первый проход инструментом. Увеличение предела текучести материала сплава АК9 происходит более чем в 1,5 раза, в то время как для сплава АК12 увеличения предела текучести не происходит. Значения предела прочности сплава АК12 повышаются на величину до $17 \%$, в то время как у сплава АК9 - на величину до 29 \%. Наиболее значительным является увеличение относительного удлинения после разрыва на 77 \% для сплава АК12 и на 113 \% для сплава АК9.

Ключевые слова: фрикционная перемешивающая обработка, алюминиево-кремниевые сплавы, прочность и пластичность металлов и сплавов, упрочнение алюминиевых сплавов

\section{1. Введение}

В настоящее время активно развиваются технологии получения и модификации металлов и сплавов, основанные на адгезионно-диффузионном фрикционном взаимодействии [1]. К ним относятся сварка трением с перемешиванием [2], фрикционная перемешивающая обработка [3] и фрикционная перемешивающая аддитивная технология [4]. Данные технологии позволяют получать изделия из алюминиевых, медных, титановых сплавов с высокой производительностью и высокими механическими свойствами полученных изделий [5-7]. Одним из возможных применений таких технологий является получение изделий из легких и прочных алюминиевых сплавов с модифицированной и упрочненной структурой поверхностного слоя методом фрикционной перемешивающей обработки [8]. В этом случае воз- 
можно как получение упрочненной структуры поверхностного слоя за счет измельчения зеренной структуры на 85-96 \% [9 ,10, 11], так и формирование композиционного материала с металлической матрицей с введением в объем обрабатываемого материала порошковых частиц $\mathrm{SiC}$ [9]; $\mathrm{Ti}_{3} \mathrm{AlC}_{2}$ [12]; $\mathrm{TiO}_{2}$ [13]; $\mathrm{Al}_{2} \mathrm{O}_{3}$ [14]; $\mathrm{NbC}$ [15], углеродных нанотрубок [16, 17] и т. д. В результате возможно получение изделий с повышенными трибологическими, механическими свойствами и коррозионной стойкостью.

Варьирование таких параметров, как число проходов ФПО, скорость вращения инструмента и скорость перемещения, приводят к неоднозначным результатам и зависят от марки алюминиевого сплава. С увеличением скорости вращения инструмента происходит увеличение среднего размера зерна и снижение прочности. В работе Н. Zhao и соавторов [10] исследовано влияние скорости вращения инструмента в диапазоне 300-1200 об/мин при однократном и многократном проходах. Показано, что средний размер зерна в зоне перемешивания в алюминиевом сплаве АД31 уменьшился в 16-26 раз. С увеличением скорости вращения инструмента с 300 до 1200 об/мин наблюдается увеличение роста зерен с 5 мкм до 8 мкм при однократном проходе и увеличение роста зерен с 8,5 до 9,7 мкм при двукратном проходе [10]. Однако несмотря на то, что ФПО привела к значительному измельчению зерен, некоторые значения механических свойств снизились по сравнению с базовым сплавом. Подобные результаты наблюдаются в работах [18]. При фиксированном значении скорости вращения инструмента (1025 об/мин) и варьировании скорости перемещения (30-150 мм/мин) также наблюдается увеличение среднего размера зерна и снижение прочностных характеристик. K.N. Ramesh и др. [19] исследовали алюминиевый сплав АМг4 при однократном и двенадцатикратном (прерывном и непрерывном) проходах ФПО с различной скоростью перемещения. Было показано, что при однократном и многократном проходах со скоростью перемещения 30 мм/мин достигаются наилучшие результаты по структуре и свойствам сплава. Дальнейшее увеличение скорости перемещения приводит сначала к увеличению среднего размера зерен, а затем к его уменьшению, повышается пластичность и снижается прочность сплава.

Среди алюминиевых сплавов для изделий с потенциально высокими трибологическими свойствами могут применяться алюминиево-кремниевые сплавы. Такие сплавы хорошо подходят для получения литых деталей различной конфигурации и изготовления деталей сложной формы методами аддитивных технологий. При этом в настоящее время в литературе имеется недостаточное количество данных о взаимосвязи, формируемой после обработки, и исходной структуры данных сплавов, определяемой содержанием кремния. Также, не имеется широкого спектра данных по влиянию на структурно-фазовое состояние таких материалов многопроходной фрикционной перемешивающей обработки (до четырех проходов инструментом), требуемой для получения материалов с равномерно распределенными в объеме частицами упрочняющих фаз. Цель настоящей работы - исследование структуры и свойств литых алюминиево-кремниевых сплавов с доэвтектической структурой (АК9) и эвтектической структурой (АК12), после фрикционной перемешивающей обработки от одного до четырех проходов инструментом.

\section{2. Материал и методика}

Образцы из сплавов АК9 и АК12 были получены в состоянии поставки в виде чушек. Для последующей обработки образцы были разрезаны на пластины (толщиной 6 мм, шириной 80 мм и длиной 300 мм) с использованием электроэрозионного станка DK 7750. После очистки и шлифовки полученных пластин производилась обработка по схеме (рис. 1). В заготовку 1 внедрялся вращающийся инструмент 2 и совершал продольное перемещение вдоль линии обработки с образованием модифицированного на глубину 3 мм поверхностного слоя 4. Обработка различных образцов производилась в 1-4 прохода инструмента по одной и той же области. Длина прохода составляла 80 мм. Сплавы АК9 и АК12 обрабатывали по заранее по- 
добранным параметрам при скорости вращения инструмента 750-800 об/мин, усилии прижима 750-800 кг и скорости продольного перемещения 90 мм/мин. Из полученных таким способом образцов, вырезали металлографические шлифы 3 и лопатки для механических испытаний 5. Металлографическую съемку производили на оптическом микроскопе Альтами MET 1C. Механические испытания на растяжение проводили на универсальной испытательной машине УТС 110М.

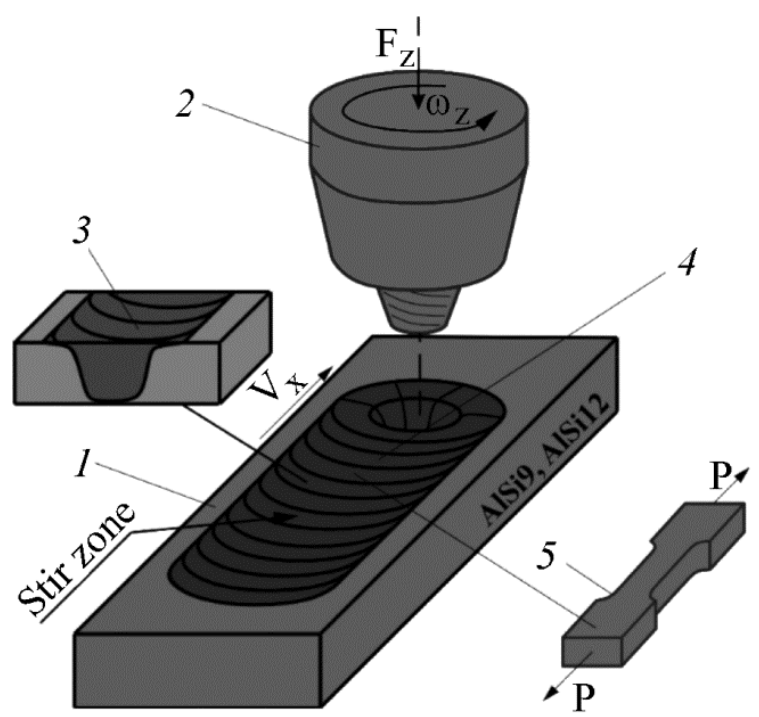

Рис. 1. Схема процесса проведения фрикционной перемешивающей обработки и вырезки образцов для механических испытаний и структурных исследований

\section{3. Результаты и обсуждение}

Макроструктура образцов сплава АК12 после 1- (a), 2- (б), 3- (в) и 4-го (2) проходов инструментом вдоль линии обработки представлена на рис. 2. В структуре образцов выделяется начиная с 1-го прохода инструментом зона перемешивания (ЗП), зона основного металла $(\mathrm{OM})$ и зона термомеханического влияния (ЗТMB) с наступающей $(\mathrm{HC})$ и отступающей (OC) стороны. Макроструктура образцов после обработки является достаточно типичной для обработки алюминиевых сплавов. В зоне обработки отсутствуют поры, которые в большом количестве присутствуют в основном металле, что свидетельствует об уплотнении материала в зоне обработки и сдавливании пор. В зоне перемешивания пластинки кремния разрушаются с образованием отдельных частиц различного размера.

При большем увеличении видно, что в материале исходного сплава АК12 частицы кремния в основном распределены в виде вытянутых пластинок (рис. $3 a-8$ ). Только отдельные частицы кремния находятся в форме угловатых частиц с формой близкой к равноосной. В зоне термомеханического влияния с отступающей стороны (рис. $32-e$ ) можно выделить наличие частично разрушенных пластинок кремния, при приближении к зоне перемешивания размер таких частиц уменьшается. В зоне перемешивания форма большинства частиц приближается к равноосной (рис. $3 \nsim-u$ ). Лишь у небольшого количества частиц форма остается вытянутой. С наступающей стороны зона термомеханического влияния менее протяженная и изменение размеров частиц кремния в ней происходит скачкообразно (рис. $3 \kappa-$ м). По контуру зоны перемешивания частицы кремния оконтуривают течение материала при обработке. 


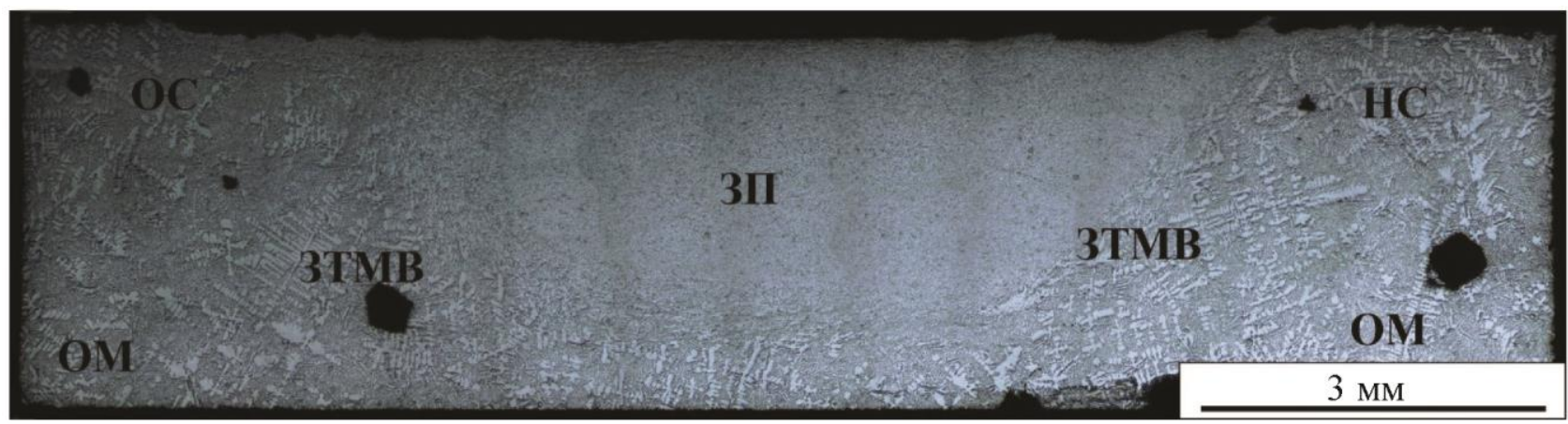

$a$

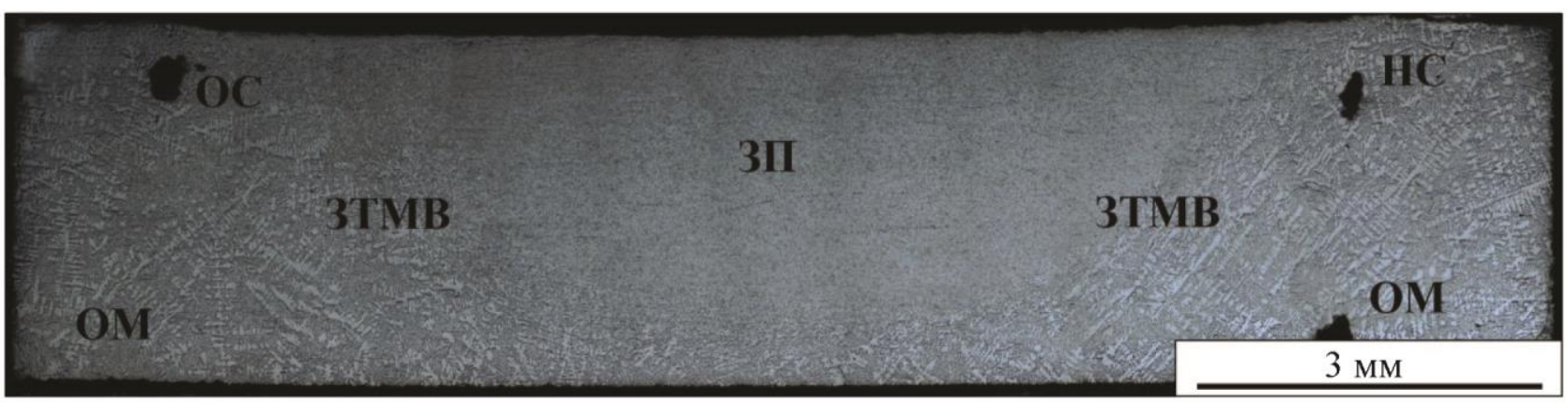

6

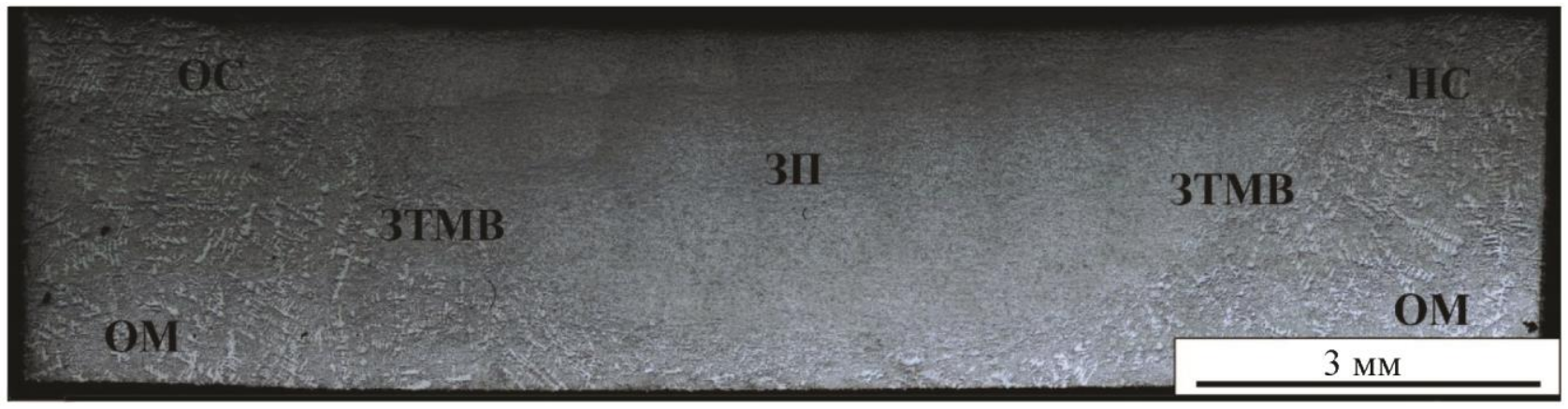

B

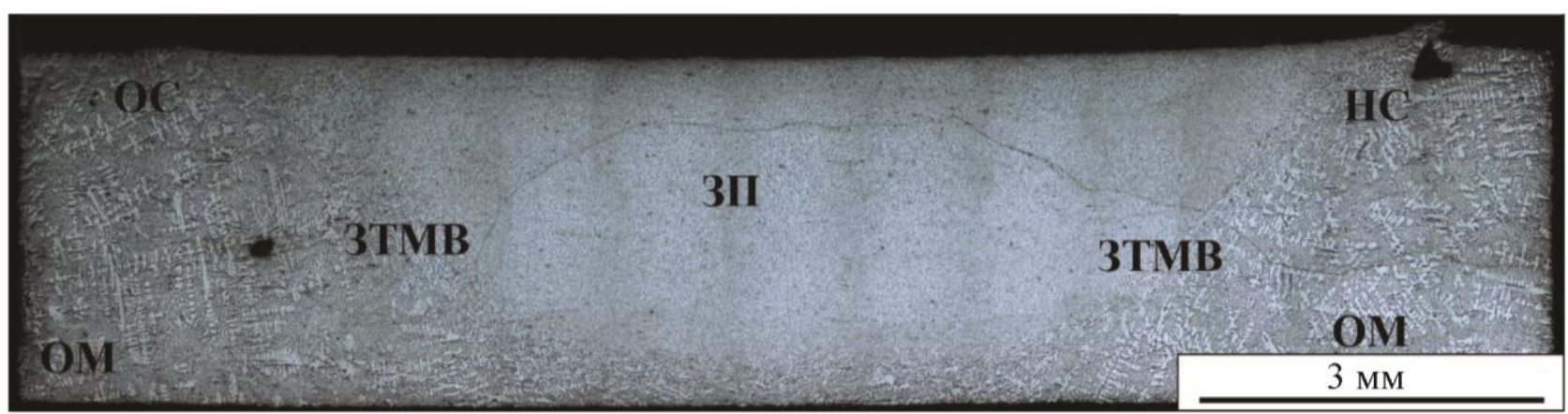

2

Рис. 2. Макроструктура образцов алюминиевого сплава АК12 после 1- (a), 2- (б), 3- (в) и 4-го (2) проходов инструментом вдоль линии обработки 


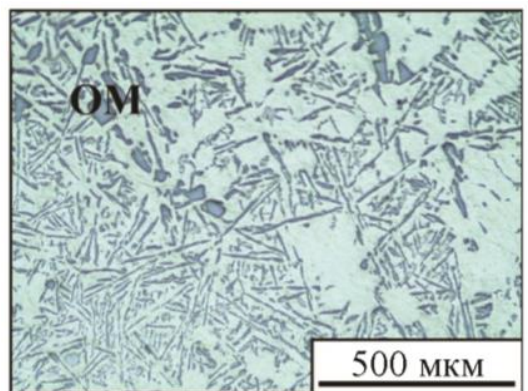

$a$

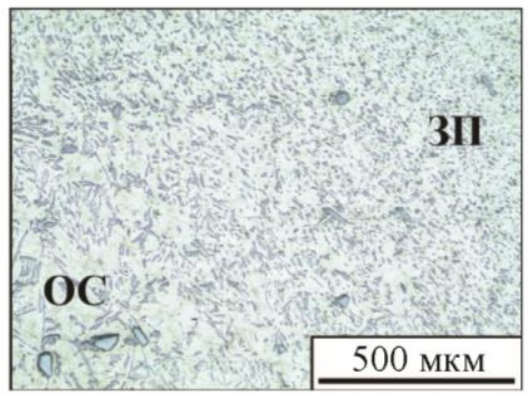

2

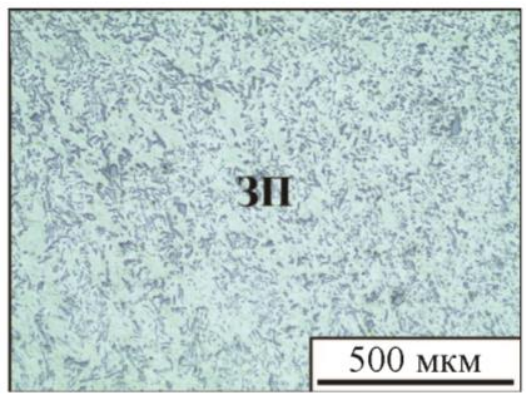

Ж

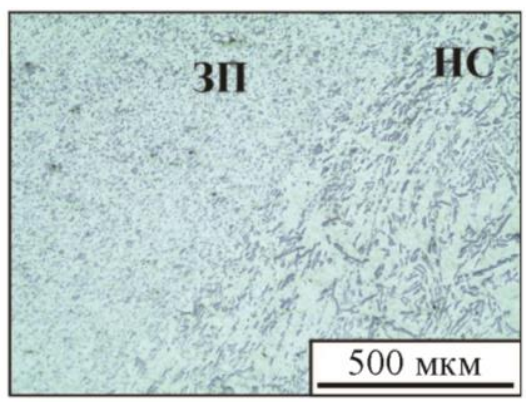

$\kappa$

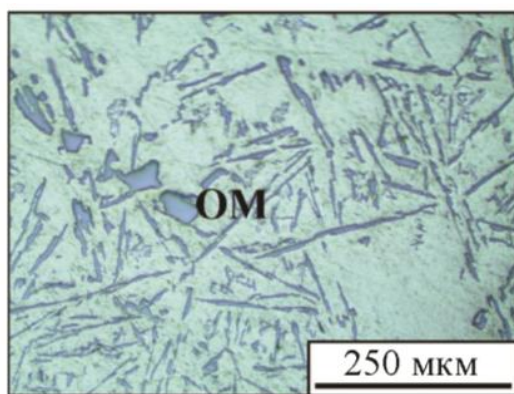

б

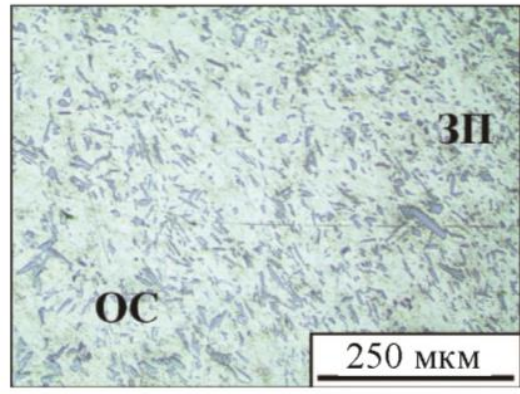

$\partial$

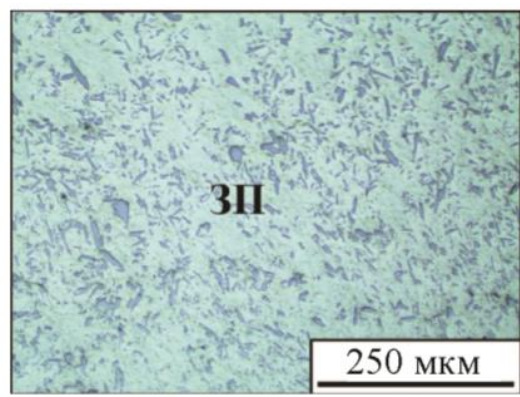

3

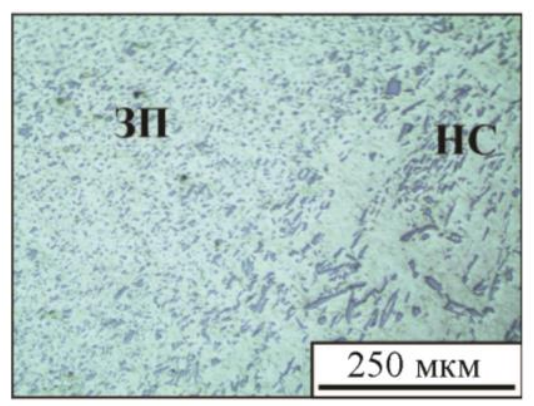

$\pi$

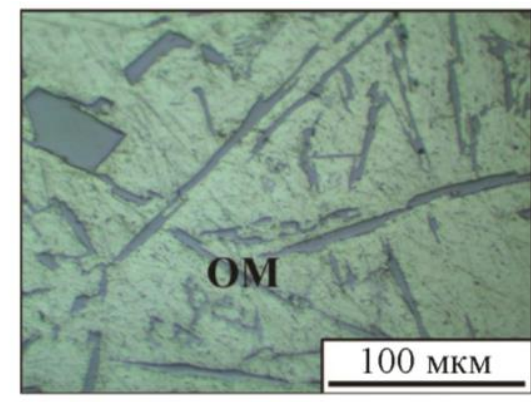

B

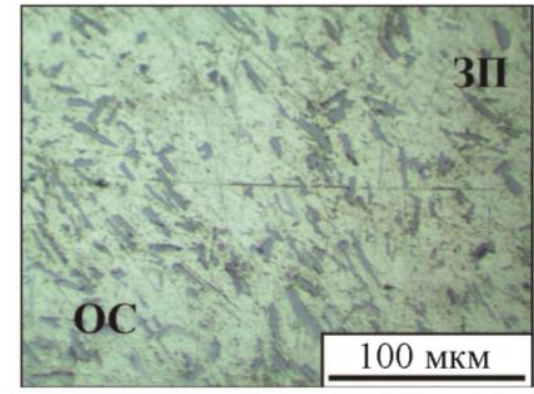

$e$

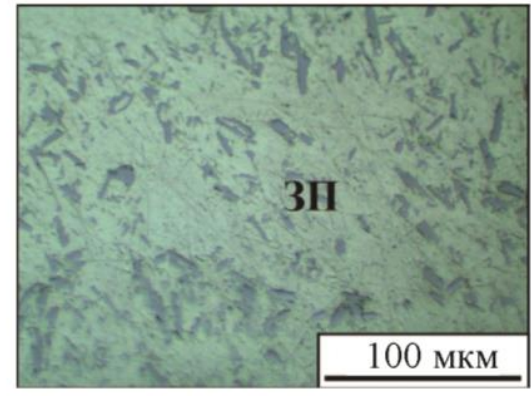

$u$

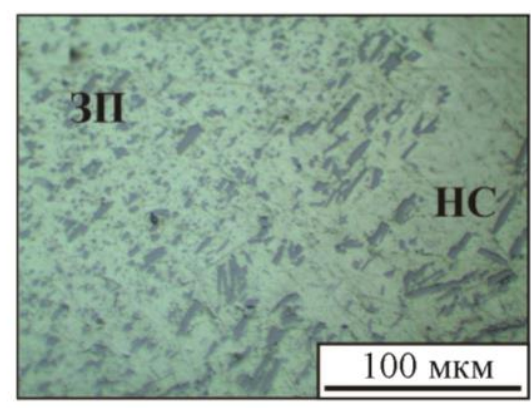

M

Рис. 3. Строение основных структурных зон сплава АК12 после однопроходной обработки: основной металл $(a-b)$; зона термомеханического влияния с отступающей стороны $(2-e)$; зона перемешивания $(ж-u)$; зона термомеханического влияния с наступающей стороны $(\kappa-M)$

При обработке от одного до четырех проходов инструментом вдоль линии обработки происходит постепенное измельчение размера частиц в зоне перемешивания (рис. 4). После одного прохода инструментом по образцу (рис. 4 a) размер частиц кремния уменьшается с 7,5 мкм в основном металле до 4,1 мкм в зоне перемешивания. При этом в зоне переме- 
шивания присутствуют также и крупные частицы, размером до 10 мкм. После двух проходов инструментом вдоль линии обработки размер частиц уменьшается до 3,7 мкм (рис. 4 б), после трех проходов - до 3,5 мкм (рис. 4 в), а после четырех - до 3,4 мкм (рис. 4 г). Таким образом, после одного прохода инструментом размер частиц уменьшается на $45 \%$, после двух - на $10 \%$, после трех - на $5 \%$ и после четырех проходов - на $3 \%$. Аналогичные данные по структуре образцов сплава АК12 после фрикционной перемешивающей обработки были получены ранее в работе [20]. В результате можно установить, что в процессе фрикционной перемешивающей обработки размер частиц наиболее существенно изменяется после первого прохода инструментом, что должно также проявляться в изменении значений механических свойств.

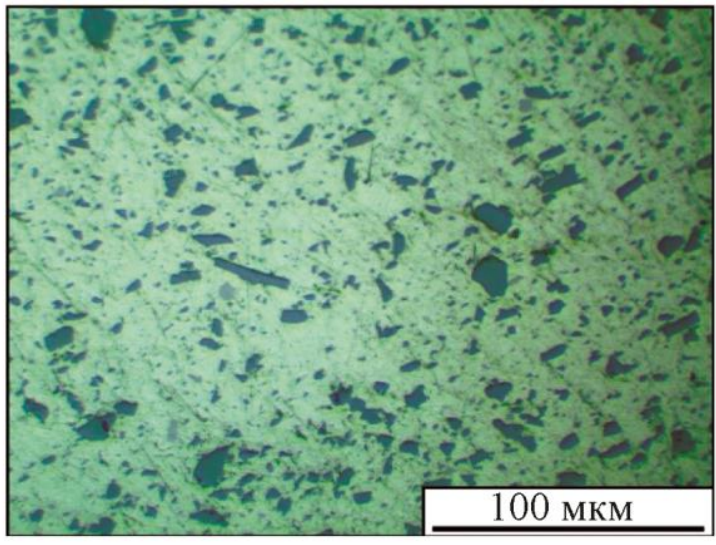

$a$

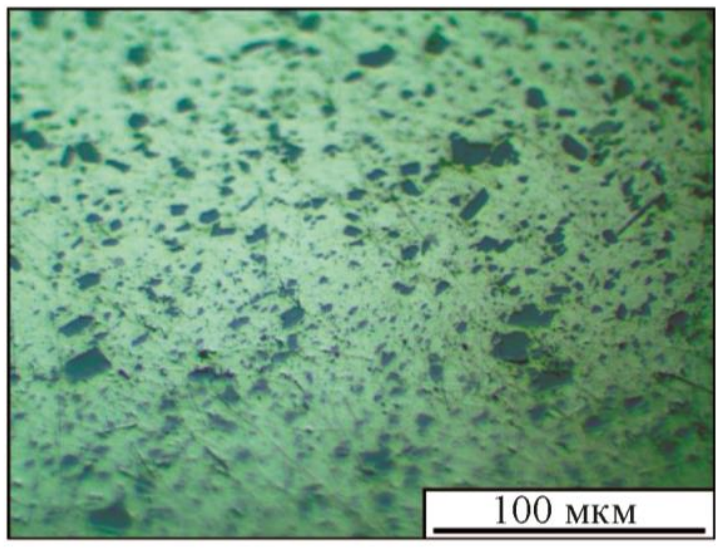

B

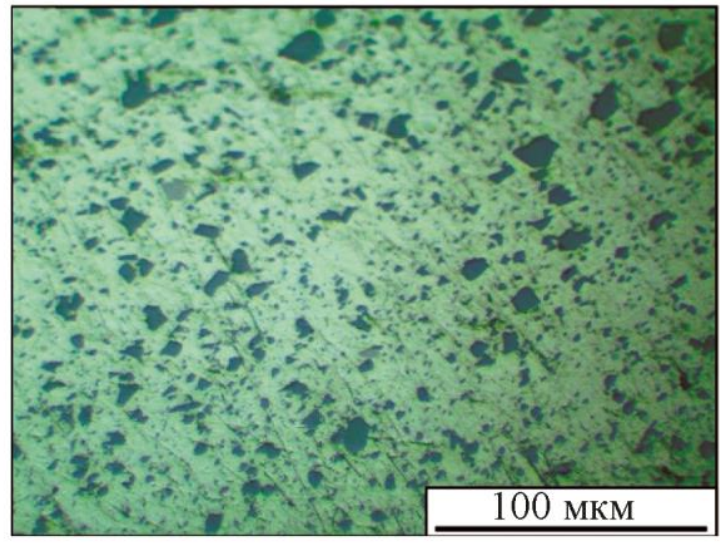

$\sigma$

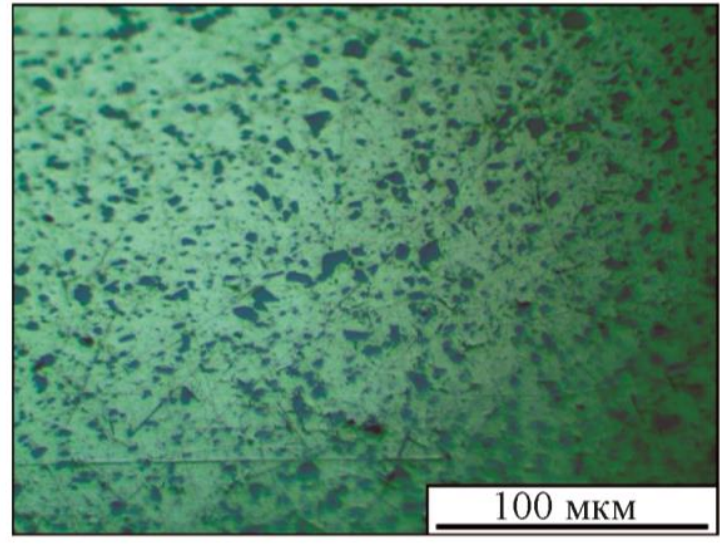

Рис. 4. Структура зоны перемешивания сплава АК12 после 1- (a), 2- (б), 3- (в) и 4-го (2) проходов инструментом вдоль линии обработки

Диаграммы напряжение-деформация образцов основного металла и материала сплава АК12 после обработки в 1-4-м проходах инструментом приведены на рис. 5. Наименьшая прочность и пластичность характерны для образцов основного металла. Из обработанных образцов наименьшая прочность у образцов после 1-го прохода инструментом (рис. $5 ; 4,1)$. При этом прочность и пластичность всех образцов существенно превышает прочность образцов до обработки. Закономерности пластической деформации образцов достаточно близки. По достижении условного предела текучести следует достаточно продолжительная стадия пластической деформации до достижения временного сопротивления, после чего происходит переход к непродолжительной стадии с практически 
постоянными значениями нагружающего усилия и последующему разрушению. В процессе деформации образцов реализуется также эффект прерывистой текучести, связываемый в работах с эффектом Портевена-Ле Шателье [21].

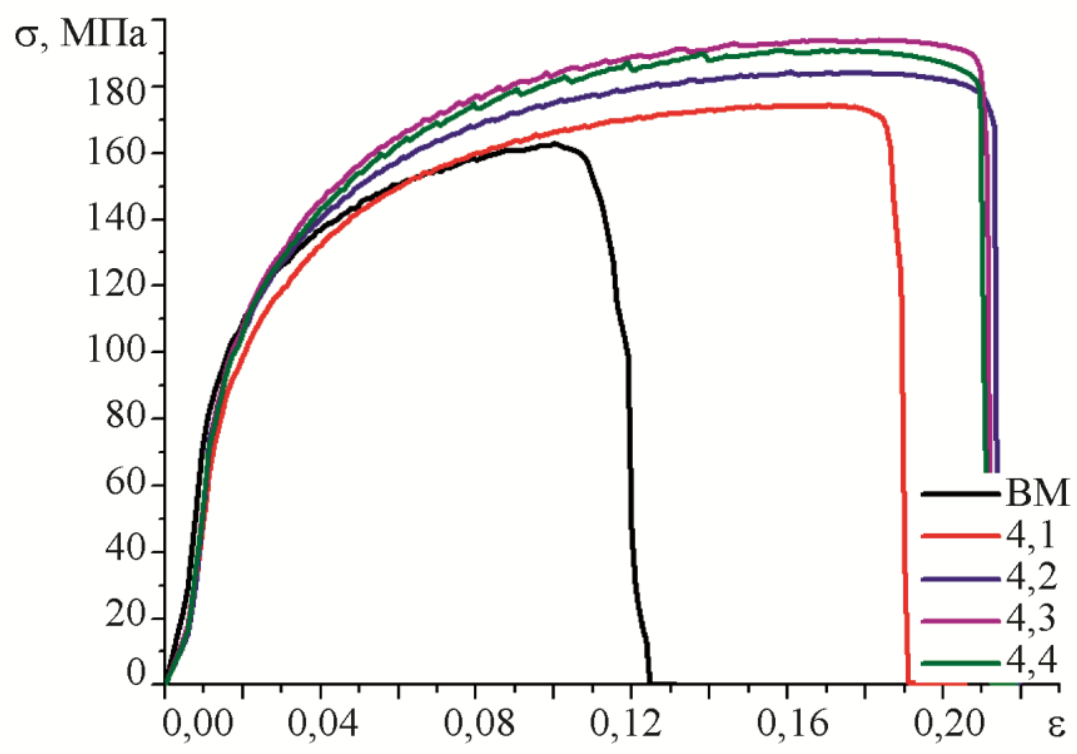

Рис. 5. Диаграммы напряжение-деформация образцов алюминиево-кремниевого сплава АК12 после 1- (4,1), 2- (4,2), 3- (4,3) и 4-го (4,4) проходов инструментом;

ВМ - основной металл

Таким образом, проведение многопроходной фрикционной перемешивающей обработки алюминиевого сплава АК12 позволило повысить временное сопротивление на 17 \%, а пластичность - более чем в два раза. Условный предел текучести при этом практически не изменяется.

Обработка образцов сплава АК9 приводит к образованию аналогичной структуры, описанной ранее для сплава АК12. Макроструктура образцов после 1-4-го проходов инструментом вдоль линии обработки представлена на рис. 6. Отличия в базовой структуре сплавов не определяют существенных отличий в организации макроструктуры зоны обработки. Так как обработка производилась одинаковым инструментом, геометрические параметры зоны перемешивания и зон термомеханического влияния не изменялись. В структуре также характерно измельчение частиц кремния в зоне перемешивания.

При большем увеличении металлографические изображения основных структурных зон области обработки приведены на рис. 7. Зона основного металла представлена крупными дендритами алюминия с располагающимися в междендритном пространстве эвтектикой на основе алюминия и пластин кремния (рис. $7 a-8$ ). В зоне термомеханического влияния структура четко выделяет направление течения материала по контуру инструмента при обработке (рис. 7 2-e). В отличии от сплава АК12 в данном случае строение зоны смешанное и представлено как деформированными и вытянутыми остатками дендритов алюминия, так и деформированными и частично разрушенными частицами кремния. В зоне перемешивания структура представляет собой аналогично описанной для сплава АК12 мелкодисперсную смесь алюминия и частиц кремния (рис. 7 ж-u). Зона термомеханического влияния с наступающей стороны также характеризуется резкой границей между зоной перемешивания и основным металлом (рис. $7 \kappa-м$ ). 


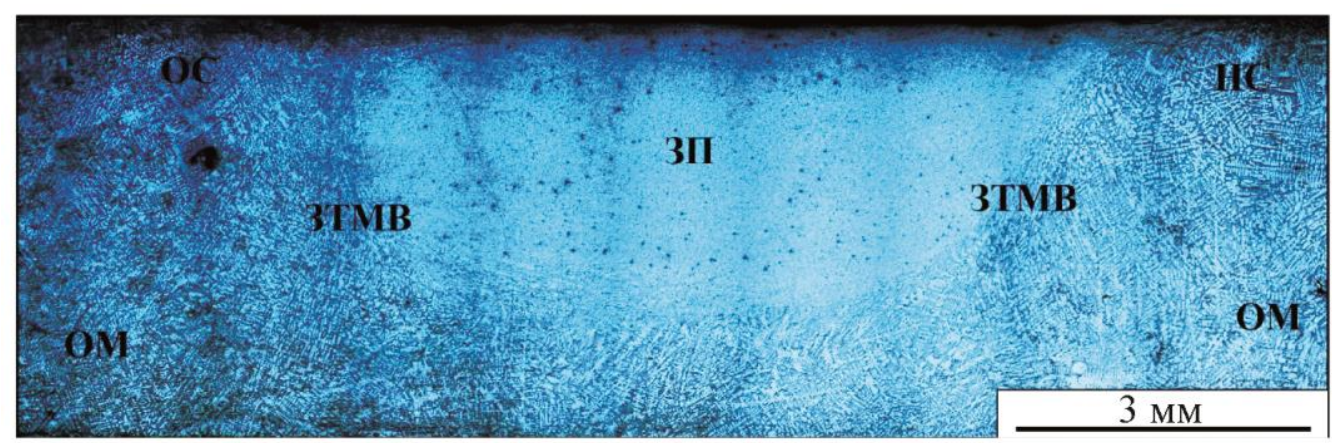

$a$

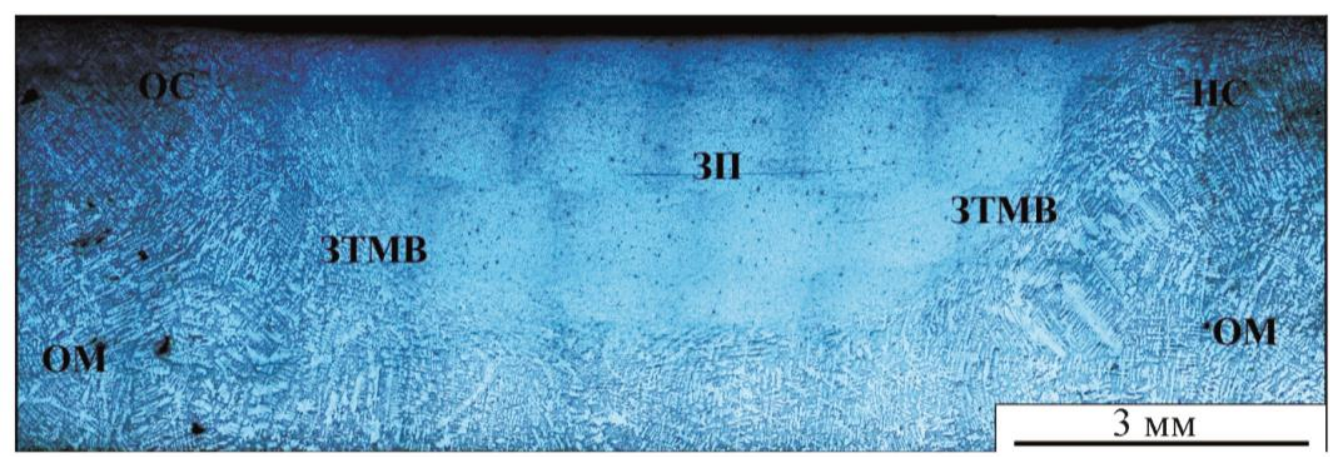

$\sigma$

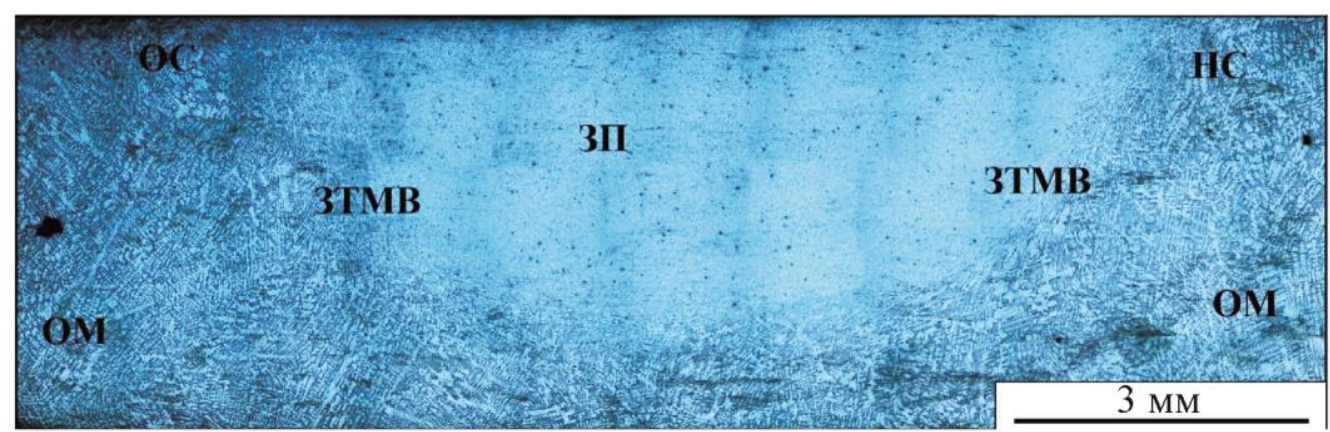

B

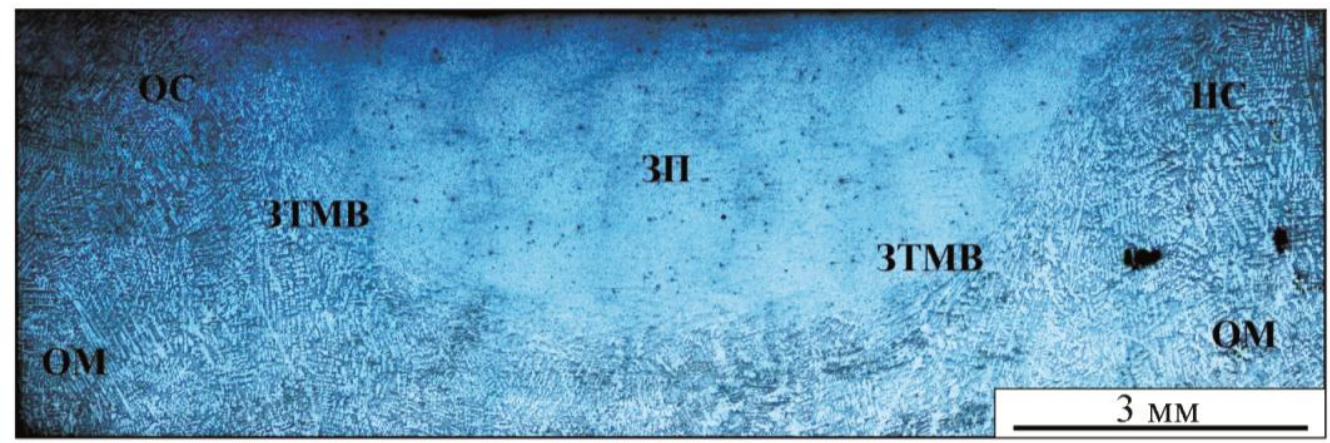

2

Рис. 6. Макроструктура образцов алюминиевого сплава АК9 после 1- (a), 2- (б), 3- (в) и 4-го (2) проходов инструментом вдоль линии обработки 


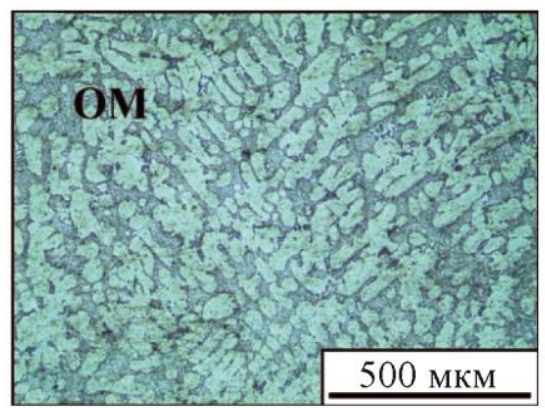

$a$

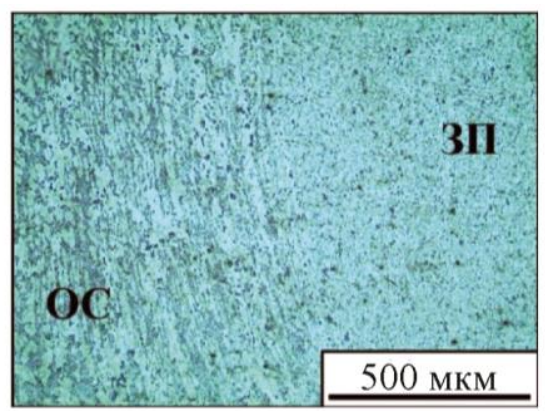

2

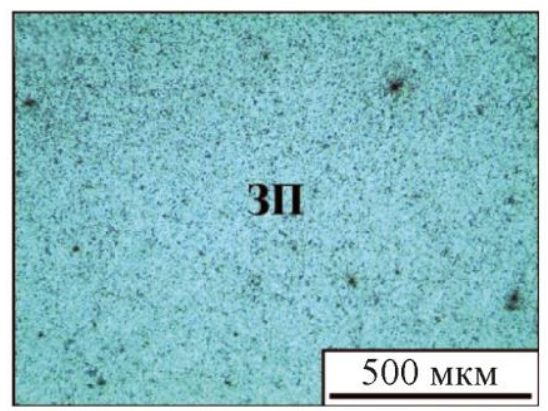

ЖC

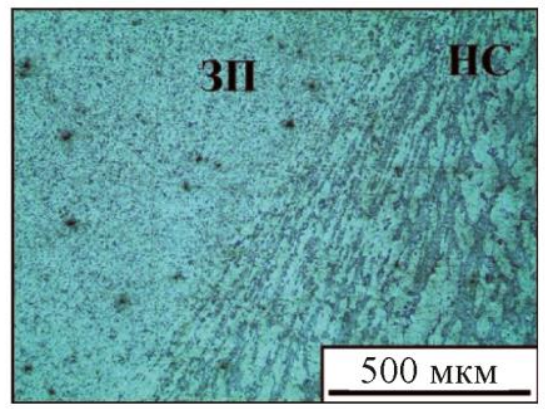

$\kappa$

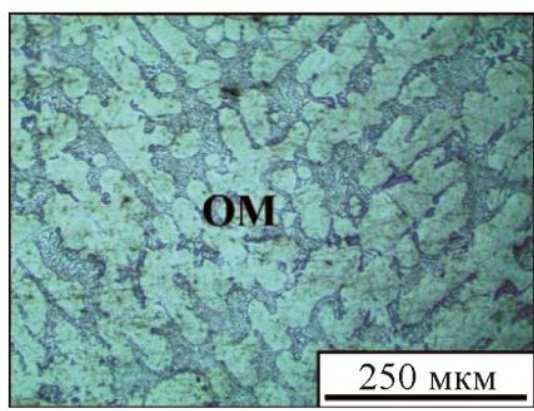

б

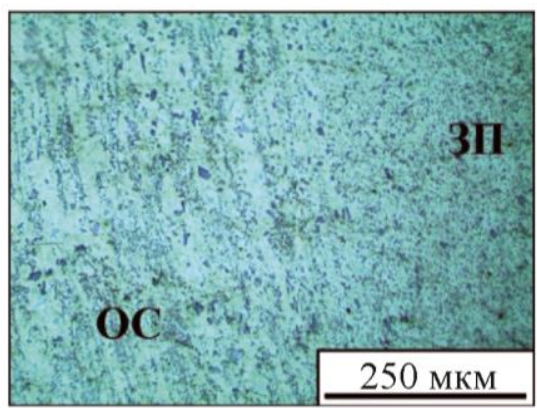

$\partial$

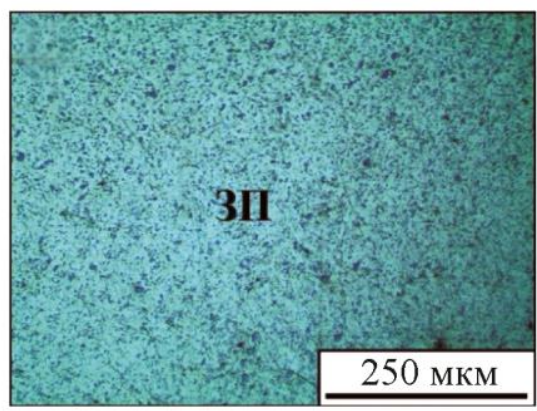

3

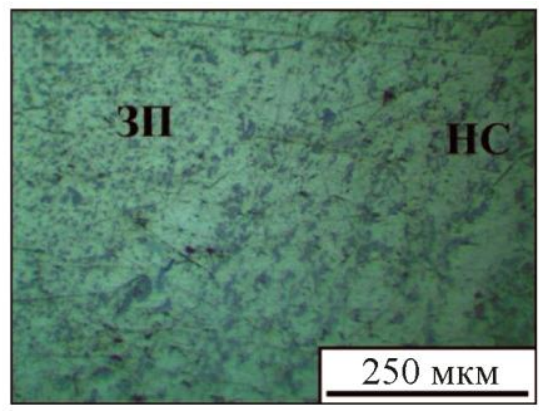

$\pi$

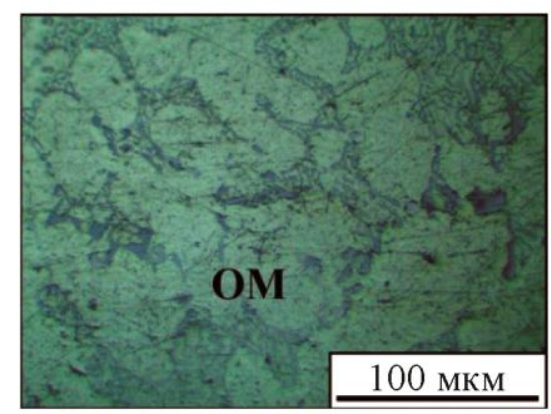

B

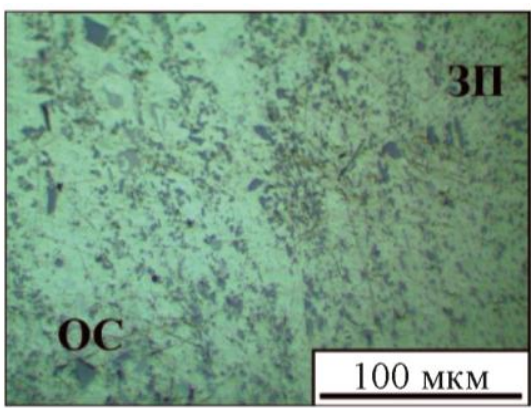

e

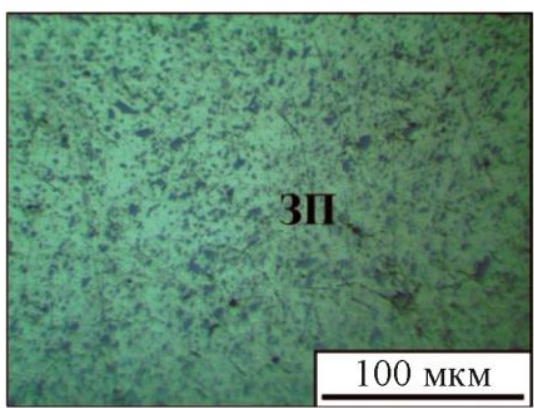

$u$

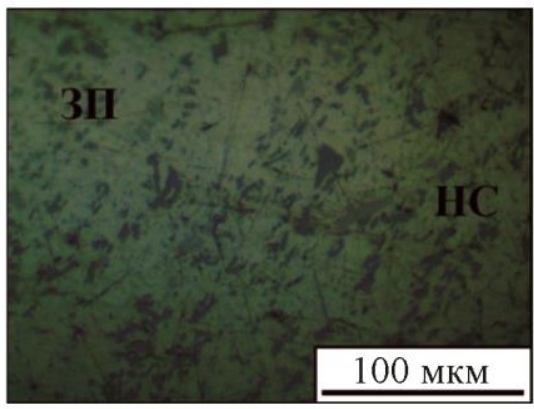

M

Рис. 7. Строение основных структурных зон сплава АК9 после однопроходной фрикционной перемешивающей обработки: основной металл $(a-b)$; зона термомеханического влияния с отступающей стороны (2-e); зона перемешивания (ж-u); зона термомеханического влияния с наступающей стороны $(\kappa-M)$

При увеличении количества проходов инструментом вдоль линии обработки для сплава АК9 существенных изменений в зоне перемешивания не обнаружено (рис. 8). После 1-го прохода (рис. 8 a) размер частиц кремния уменьшается с 5,4 мкм до 2,9 мкм. После 2-го - до 2,8 мкм 
(рис. 8 б). После 3-го до 2,3 мкм (рис. 8 в). После 4-го не изменяется (рис. 8 г). Таким образом, размер частиц кремния в образцах наиболее интенсивно изменяется после первого прохода инструментом вдоль линии обработки, аналогично наблюдаемому изменению при обработке сплава АК12. Меньший исходный размер пластин кремния в данном случае обусловливает меньший размер частиц в зоне перемешивания.

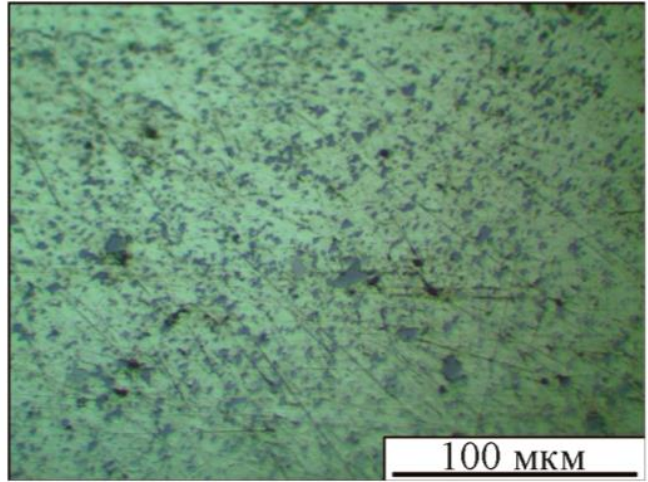

$a$

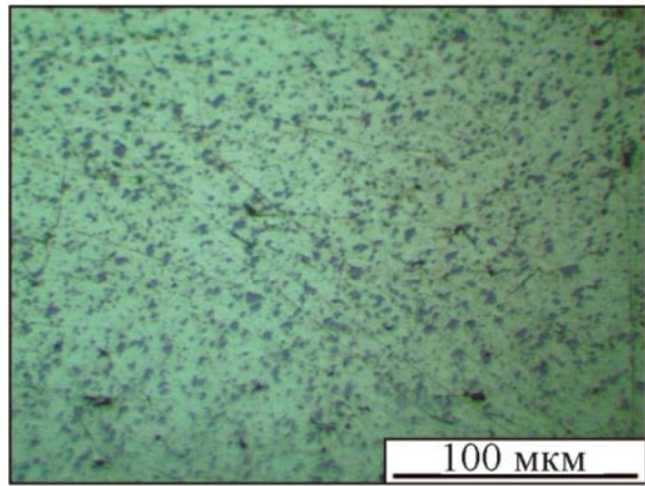

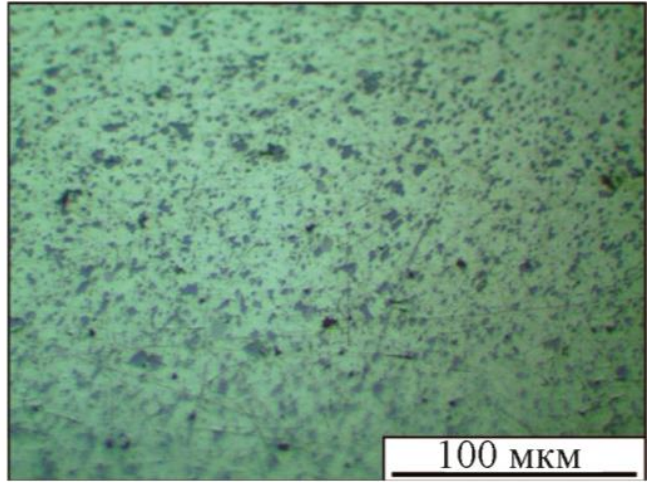

б

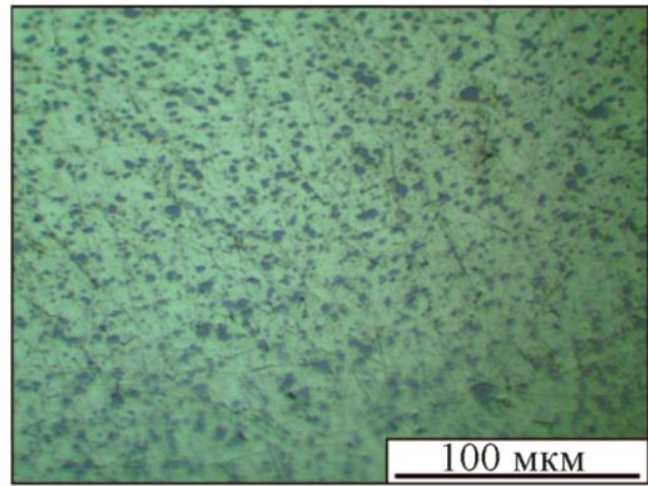

Рис. 8. Структура зоны перемешивания сплава АК9 после 1- (a), 2- (б), 3- (в) и 4-го (2) проходов инструментом вдоль линии обработки

Основное отличие деформационного поведения образцов сплава АК9 после фрикционной перемешивающей обработки от аналогичных образцов сплава АК12 заключается в существенном влиянии обработки на значения условного предела текучести по сравнению с образцами основного металла (рис. 9). В то время как у образцов основного металла условный предел текучести находится на уровне 95 МПа, в обработанных образцах он может достигать 140 МПа. Характерной для образцов сплава АК12 прерывистой текучести в данном материале не обнаружено. Влияние обработки на пластичность материала аналогичное, но увеличение пластичности несколько ниже $(1,85$ раза, по сравнению с 2,13 раза у сплава АК12). Повышение пластичности после обработки связано прежде всего с разрушением пластин кремния и измельчением структурных составляющих. Увеличение временного сопротивления наиболее интенсивно после 1-го прохода - $79 \%$. После 2-го прохода прочность повышается менее чем на $3 \%$; после 3-го - снижается на $3 \%$, а после 4-го повышается на те же $3 \%$. Связано это с тем, что структура материала после 2-4-го проходов изменяется не так существенно, как после 1-го, когда разрушаются изначально крупные и вытянутые пластинки кремния. 


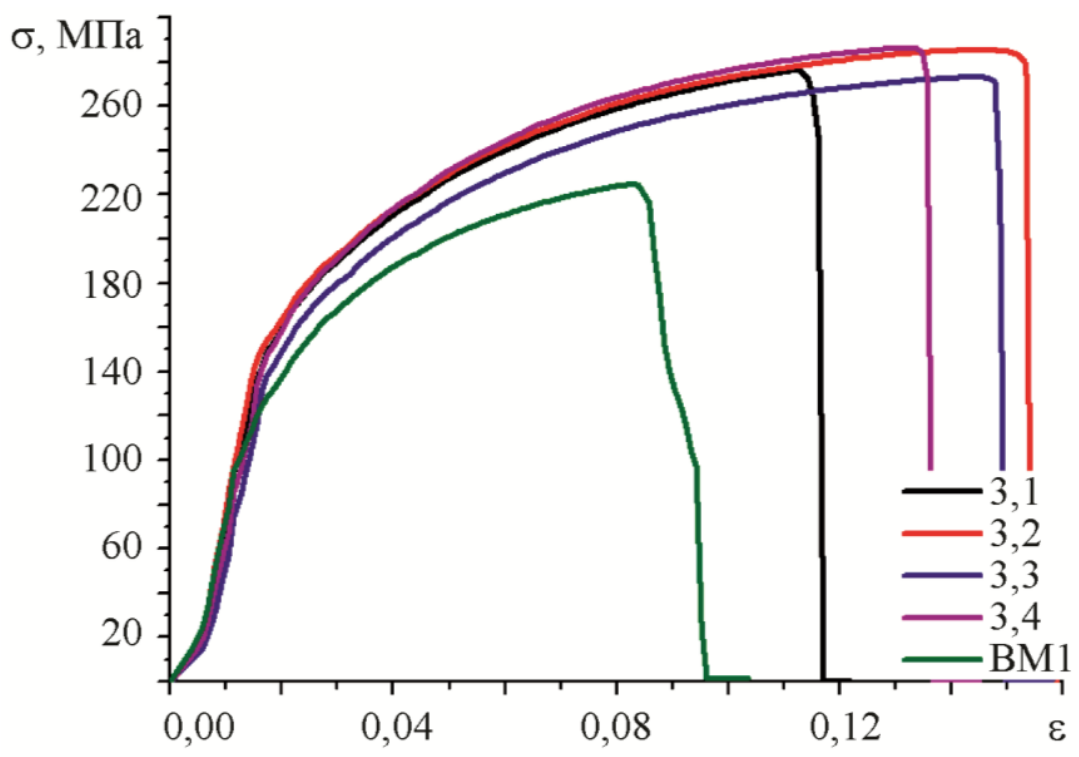

Рис. 9. Диаграммы напряжение-деформация образцов алюминиево-кремниевого сплава АК9 после 1- $(3,1), 2$ - $(3,2), 3$ - $(3,3)$ и 4-го $(3,4)$ проходов инструментом; ВМ1 - основной металл

\section{4. Заключение}

Проведенные исследования показывают, что после фрикционной перемешивающей обработки алюминиево-кремниевых сплавов АК9 и АК12 происходит разрушение пластинок кремния с образованием мелкодисперсной структуры зоны перемешивания. Наиболее существенные структурные изменения в образцах происходят после первого прохода инструментом вдоль линии обработки, хотя в целом многопроходная обработка позволяет получать более мелкодисперсную структуру зоны перемешивания. Помимо формирования мелкодисперсной и однородной структуры обработка приводит также и к удалению из объема материала пор, в большом количестве присутствующих в отливках. С точки зрения влияния обработки на совокупность механических свойств исследованных в работе материалов (рис. 10) можно выделить также положительные изменения.

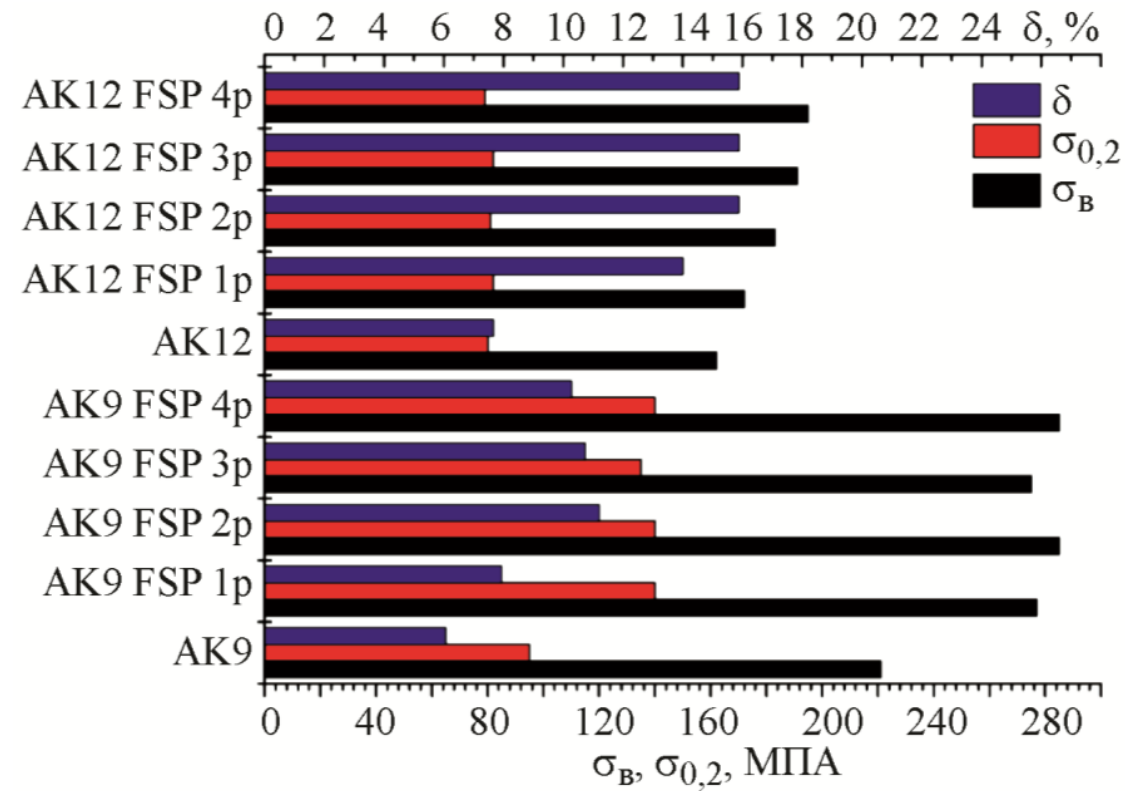

Рис. 10. Механические свойства сплавов АК9 и АК12 после многопроходной фрикционной перемешивающей обработки 
Временное сопротивление образцов наиболее существенно изменяется при обработке сплава АК9, но для сплава АК12 более явным является влияние количества проходов инструментом вдоль линии обработки на значение временного сопротивления. Влияние обработки на условный предел текучести у сплава АК12 практически отсутствовало, в то время как для сплава АК9 было достаточно ощутимым, хотя и не проявлялось влияния количества проходов на механические свойства. Применительно к сплаву АК12 наибольшее влияние обработка оказала на увеличение пластичности, приводя к более чем двукратному ее росту. При этом для сплава АК9 увеличение пластичности было также существенным, но на несколько меньшую величину. Таким образом, обработка изделий из алюминиево-кремниевых литых сплавов потенциально обладает актуальностью для применения с целью упрочнения поверхностного слоя или как метод для формирования композиционных материалов с металлической матрицей.

\section{Благодарность}

Работа выполнена в рамках государственного задания ИФПМ СО РАН, тема номер FWRW-2021-0012.

\section{Литература}

1. Закономерности формирования материалов с композитной структурой с использованием аддитивной электронно-лучевой технологии, сварки трением с перемешиванием и фрикционной перемешивающей обработки / Т. А. Калашникова, А. В. Гусарова, А. В. Чумаевский, Е. О. Княжев, М. А. Шведов, П. А. Васильев // Обработка металлов (технология - оборудование инструменты). - 2019. - Т. 21, № 4. - С. 94-112.

2. Mishra R. S., Ma Z. Y. Friction stir welding and processing // Materials Science and Engineering: R: Reports. - 2005. - Vol. 50. - P. 1-78. - DOI: 10.1016/j.mser.2005.07.001.

3. Li K., Liu X., Zhao Y. Research Status and Prospect of Friction Stir Processing Technology // Coatings. - 2019. - Vol. 9, No. 2. - P. 1-14. - DOI: 10.3390/coatings9020129.

4. Processing-structure-property correlation in additive friction stir deposited Ti-6Al-4V alloy from recycled metal chips / P. Agrawal, R. S. Haridas, S. Yadav, S. Thapliyal, S. Gaddam, R. Verma, R. S. Mishra // Additive Manufacturing. - 2021. - Vol. 47. - P. 102259. DOI: 10.1016/j.addma.2021.102259.

5. A Review of Friction Stir Processing of Structural Metallic Materials: Process Properties, and Method / A. P. Zykova, S. Y. Tarasov, A. V. Chumaevskiy, E. A. Kolubaev // Metals. - 2020. Vol. 10. - DOI: 10.3390/met10060772.

6. Ma Z. Y. Friction Stir Processing Technology: A Review // Metallurgical and Materials Transactions A. - 2008. - Vol. 39. - P. 642-658. - DOI: 10.1007/s11661-007-9459-0.

7. Towards aging in a multipass friction stir-processed AA2024 / K. N. Kalashnikov, S. Y. Tarasov, A. V. Chumaevskii, S. V. Fortuna, A. A. Eliseev, A. N. Ivanov // International Journal of Advanced Manufacturing Technology. - 2019. - Vol. 103, Nos. 5-8. - P. 2121-2132. DOI: $10.1007 / \mathrm{S} 00170-019-03631-3$.

8. Hsu C. J., Kao P. W., Ho N. J. Intermetallic-reinforced aluminum matrix composites produced in situ by friction stir processing // Materials Letters. - 2007. - Vol. 61, No. 6. - P. 1315-1318. DOI: 10.1016/J.MATLET.2006.07.021.

9. Investigating effects of process parameters on microstructural and mechanical properties of Al5052/SiC metal matrix composite fab-ricated via friction stir processing / A. Dolatkhah, P. Golbabaei, M. K. Besharati Givi, F. Molaiekiya // Materials and Design. - 2012. - Vol. 37. P. 458-464. - DOI: 10.1016/J.MATDES.2011.09.035.

10. Effect of the processing parameters of friction stir processing on the microstructure and mechanical properties of 6063 aluminum alloy / H. Zhao, Q. Pan, Q. Qin, Y. Wu, X. Su // Materials Science \& Engineering A. - 2019. - Vol. 751. - P. 70-79. - DOI: 10.1016/J.MSEA.2019.02.064. 
11. Su J.-Q., Nelson T. W., Colin S. J. Microstructure evolution during FSW/FSP of high strength aluminum alloys // Materials Science and Engineering. - 2005. - Vol. 405, iss. 1-2. P. 277-286. - DOI: 10.1016/J.MSEA.2005.06.009.

12. On the surface reinforcing of $\mathrm{A} 356$ aluminum alloy by nanolayered $\mathrm{Ti}_{3} \mathrm{AlC}_{2} \mathrm{MAX}$ phase via friction stir processing / A. Manochehrian, A. Heidarpour, Y. Mazaheri, S. Ghasemi // Surface \& Coatings Technology. - 2019. - Vol. 377. - P. 124884. - DOI: 10.1016/J.SURFCOAT.2019.08.013.

13. Jain V. K. S., Varghese J., Muthukumaran S. Effect of First and Second Passes on Microstructure and Wear Properties of Titanium Dioxide-Reinforced Aluminum Surface Composite via Friction Stir Processing // Arabian Journal for Science and Engineering. - 2019. Vol. 44. - P. 949-957. - DOI: 10.1007/S13369-018-3312-1.

14. Bourkhani Darzi R., Eivani A. R., Nateghi H. R. Through-thickness inhomogeneity in microstructure and tensile properties and tribological performance of friction stir processed AA1050-A12O3 nano-composite // Composites Part B. - 2019. - Vol. 174. - P. 107061. DOI: 10.1016/J.COMPOSITESB.2019.107061.

15. Characterization of NbC-Reinforced AA7075 Alloy Composites Produced Using Friction Stir Processing / T. Satish Kumar, G. Suganya Priyadharshini, S. Shalini, K. Krishna Kumar, R. Subramanian // Transactions of the Indian Institute of Metals. - 2019. - Vol. 72, No. 6. P. 1593-1596. - DOI: 10.1007/s12666-019-01566-7.

16. Effect of filler material and post process ageing treatment on microstructure, mechanical properties and wear behaviour of friction stir processed AA 7075 surface composites / H. A. Deore, J. Mishra, A. G. Rao, H. Mehtani, V. D. Hiwarkar // Surface \& Coatings Technology. - 2019. Vol. 374. - P. 52-64. - DOI: 10.1016/J.SURFCOAT.2019.05.048.

17. Effects of energy input during friction stir processing on microstructures and mechanical properties of aluminum/carbon nanotubes nanocomposites / S. Zhang, G. Chen, J. Wei, Y. Liu, R. Xie, Q. Liu, S. Zeng, G. Zhang, Q. Shi // Journal of Alloys and Compounds. - 2019. - Vol. 798. P. 52-530. - DOI: 10.1016/J.JALLCOM.2019.05.269.https://doi.org/10.1016/j.jallcom.2019.05.269

18. Abrahams R., Mikhail J., Fasihi P. Effect of friction stir process parameters on the me-chanical properties of 5005-H34 and 7075-T651 aluminium alloys // Materials Science \& Engineering A. 2019. - Vol. 751. - P. 363-373. - DOI: 10.1016/J.MSEA.2019.02.065.

19. Ramesh K. N., Pradeep S., Pancholi V. Multipass Friction-Stir Processing and its Effect on Mechanical Properties of Aluminum Alloy 5086 // Metallurgical and Materials Transactions A. 2012. - Vol. 43. - P. 4311-4319. - DOI:10.1007/s11661-012-1232-3.

20. Friction Stir Processing Regularities of Cast Aluminum Alloy AlSi12 / D. V. Indoitu, A. V. Gusarova, A. P. Zykova, T. A. Kalashnikova, A. V. Chumaevskii, D. A. Gurianov, V. A. Beloborodov // Journal of Physics: Conference Series. - 2021. - Vol. 1989, No. 1. P. 012030. - DOI: 10.1088/1742-6596/1989/1/012030.

21. Abbadi M., Hähner P., Zeghloul A. On the characteristics of Portevin-Le Chatelier bands in aluminum alloy 5182 under stress-controlled and strain-controlled tensile testing // Materials Science and Engineering A. - 2002. - Vol. 337, iss. 1-2. - P. 194-201. - DOI: 10.1016/S09215093(02)00036-9. 